\title{
Multiple Primary Cancers in North Tunisia, 2000 - 2009
}

\section{Wided Ben Ayoub ${ }^{1}$, Hajer Ben Khadhra ${ }^{1}$, Hyem Khiari', Mansour Ben Abdallah', Hamouda Boussen ${ }^{2}$, Mohamed Hsairi ${ }^{1}$}

${ }^{1}$ Department of bio statistics and epidemiology, Cancer Registry North of Tunisia, Salah Azaiz Institute, Tunis, Tunisia. ${ }^{2}$ Department of medical oncology, Abdelrahmen Mami Hospital, Ariana, Tunisia.

\begin{abstract}
Background and Objective: To evaluate and report the frequency, epidemiologic and anatomic - clinical features of patients who developed MPM from the data of North Tunisia Cancer Registry, during the period 2000-2009. Materials and methods: From a population of 53757 new patients of the North Tunisia National Cancer Registry database presenting new cases of cancers during the period 2000-2009 in North Tunisia, we collected and analyzed those with MPMTs. We used for MPMT the international IARC diagnosis criteria are published in ICD-O Third Edition. Results: In the 53757 new cancer cases registered from 2000-2009, we collected 528 cases $(1.0 \%)$ of MPM. Mean age at diagnosis of the 1 st cancer was 61 years (22-99) and sex-ratio at 1.08 $(275 \mathrm{M} / 253 \mathrm{~F})$ while mean age at the 2 nd cancer diagnosis was 62 years (29 to 99). Among the 528 cases, the most frequent 1 st cancer site was breast in females (147 pts, 58.1\%) and urinary tract for males (56 patients, 20.4\%). In the 528 MPM cases, $321(60.8 \%)$ were synchronous and 207 cases $(39.2 \%)$ were metachronous tumors. The median time from 1 st to 2 nd cancer was 1.98 months (range $0-140$ ). The most associated 1 st -2 nd cancer sites were breast in 110 patients (43.3\%) in females and for males' urinary tract -prostate cancers (45 patients, $16.3 \%$ ). Conclusions: The coexistence of a synchronous or metachronous MPM is possible and have to be considered during pretreatment evaluation. A close follow-up should be recommended to detect second malignancies in patients treated for a 1 st cancer.
\end{abstract}

Keywords: Multiple primary malignancies- clinical features- North Tunisia

Asian Pac J Cancer Care, 3 (3), 59-63

\section{Introduction}

Multiple primary malignancies (MPM) are defined by two or more malignancies in the same patient, at diagnosis or during the evolution of cancer [1]. The occurrence of MPM is reported in $0.7 \%$ to $18 \%$ of cancer patients [2-3]. This event is probably related to therapeutic advances that prolonged survival in localized and even locally advanced tumors, increasing the risk of MPM occurrence for metachronous tumors [1-2-4]. MPM positive diagnosis is based on Warren and Gates criteria's proposed in 1932 [5] rapport airtum, 2013). Synchronous cancers are second tumors occurring simultaneously or within 6 months after the 1stmalignancy, while metachronous multiple malignancies are diagnosed more than 6 months after the initial cancer diagnosis [6-7].Our study aim's to evaluate the frequency of patients diagnosed with MPM from the database of the North Tunisia Cancer Registry
Submission Date: 06/19/2018 Acceptance Date: 08/21/2018

throughout a period of 9 years. We collected the most common types of multiple malignant tumors associations between different types of cancers and the frequency of synchronous and metachronous cancers.

\section{Materials and Methods}

We reviewed retrospectively 53757 cancer new cases from the database of the North Tunisia Cancer Registry registered between January 2000 and December 2009. We collected among the MPM's defined as the presence of at least two cancers locations, histological confirmed, with distinct histology in the two locations. We used also the recommendation for recording of International Rules for Multiple Primary Cancers given by the IARC (ICD-O Third Edition), where Two tumors of different laterality, but of the same morphology, diagnosed in paired organs (e.g. breast) should be registered separately

\section{Corresponding Author:}

Dr. Wided Ben Ayoub

Department of bio statistics and epidemiology, Cancer Registry North of Tunisia, Salah Azaiz Institute, Tunis, Tunisia.

Email: wided.benayoub@rns.tn 
unless stated to have originated from a single primary [7]. Exceptions to this rule are:

a)Tumors of the ovary (of the same morphology)

b) Wilm's tumor (nephroblastoma) of the kidney.

c) Retinoblastoma

These anatomic sites should be recorded as a single bilateral registration when they occur on both sides. The time interval to differentiate between synchronous or metachronous was taken as 6 months as reported by several authors [8-10].

Percent of tumors classified as multiple primary cancers were calculated by gender, age $(<=39,40-$ 49, 50-59, 60-69, 70-79, and 80+), and anatomic site, respectively. To evaluate whether cancers registered as multiple primary cancers were more likely to be diagnosed at an early stage, percent of local-staged tumors among multiple primary cancers was compared with that among first primary cancers. Statistical analyses were performed using The SPSS (version 15.0; SPSS Inc., Chicago, USA) statistical software in the present study. All data were expressed as the mean \pm standard deviation or number with the percentage $(\%)$. We used the ANOVA test for comparing the values of each group. A $p$ value less than 0.05 was considered statistically significant.

\section{Results}

\section{Patients Characteristics}

Over a period of 10 years, we collected 528 cases (M/F 275/253, sex-ratio 1/08) of MPM's representing $1 \%$ of the cancers during the same period. Mean age at diagnosis of the 1stcancer was61 years and median at 62 years, while mean age at the 2 nd cancer was 62 years and median at 63 years $(\mathrm{p}<0.000)$. The median ages $1 \mathrm{st}$ cancer diagnosis was 68 years $(38-85)$ for males and 54 years (22-99) for females $(\mathrm{P}<.0 .000)$

\section{Distribution according to sites}

In females, the most common site of 1 st cancer was breast (147 cases, $58.1 \%$ ) followed by gynecological (30 cases, 11, 9\%) and colorectal cancers (17case; 6, 7\%). In males (Figure 1), the predominant site was urinary

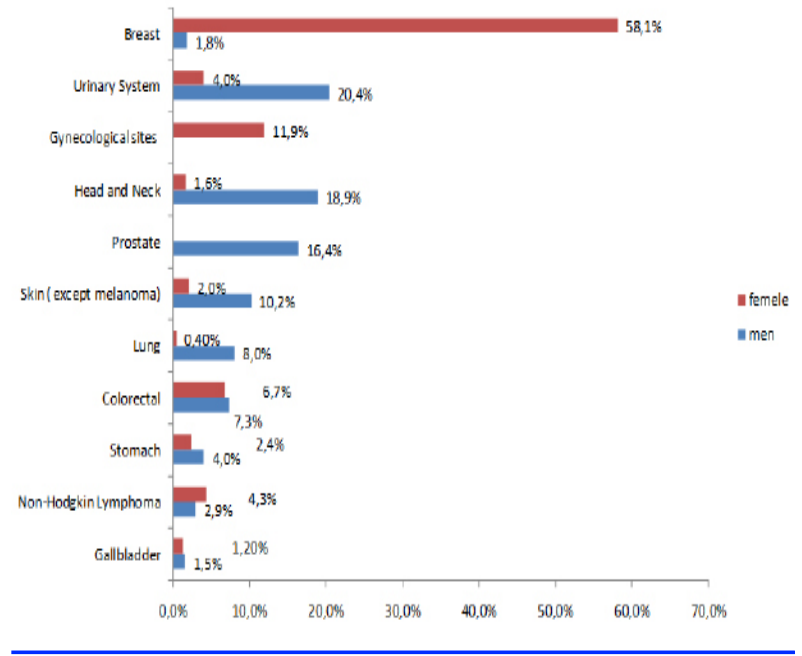

Figure1. Distribution of First Primary Cancer Site According to Gender

tract cancer ( 56 cases, 20.4\%), followed by head and neck (52cases, 18, 9\% ) and prostate cancer in 45 cases $(16,4 \%)$. The 2 nd cancer was in females, predominantly in the breast (142 patients, 55.9\%), then gynecological (43 cases, 17\%) and colorectal cancer (18cases, 4.1\%). In males (Figure 2), the 2nd most frequent cancer site is lung (56 cases, 20.4\%) followed by urinary tract cancer (53 patients, 19.3\%) and head and neck cancers (34 cases, 12, 4\%). The most frequently observed tumor pairs 1 st-2nd cancers (Figures 3 and 4) were breast-breast in females and (110 cases, $43.3 \%)$ and prostate-urinary tract ( 45 cases $16.3 \%$ ) in males.

Both primary and secondary tumors tended to be in advanced stages III and IV in $27.1 \%$ and $26.4 \%$, as well as the lung cancers (Table 1)

The mean time between 1 st and 2 nd cancer is 12.2 months (0-140). Within the 528 patients, $321(60.8 \%)$ were diagnosed with synchronous MPM (sMPM) and $207(39.2 \%)$ with metachronous or mMPM. mMPM diagnosed occurred after a mean time of 29.9 months (6-140.8) after the 1st cancer diagnosis. The sex-ratio was $0.83(\mathrm{M} / \mathrm{F} 146 / 175)$ for sMPM and 1.65(M/F 129/78) for MPM. The most frequently detected SMPM in females

Table 1. Percent of Cancers Diagnosed at Local Stage by Cancer Site and Primary Status (First primary vs. multiple primaries)

\begin{tabular}{lccc}
\hline & $\begin{array}{c}\text { (A) \% local stage among } \\
\text { first primary cancer }\end{array}$ & $\begin{array}{c}\text { (B) \% local stage among } \\
\text { second primary cancer }\end{array}$ & ratio B/A \\
\hline All sites & 27.1 & 26.4 & 0.9 \\
Head and neck & 29.6 & 28.6 & 0.9 \\
Stomac & 17.6 & 0 & 0 \\
colorectal & 16.7 & 25.8 & 1.5 \\
Lung & 0 & 0 & 1.9 \\
Skin (withoutmelanoma) & 35.5 & 67.9 & 1.12 \\
Breast & 33.3 & 37.5 & 0.9 \\
Gynecological sites & 27.6 & 26.8 & 1.1 \\
Prostate & 25.6 & 29.6 & 1.1 \\
Urinary system & 25.4 & 29.8 & \\
\hline
\end{tabular}




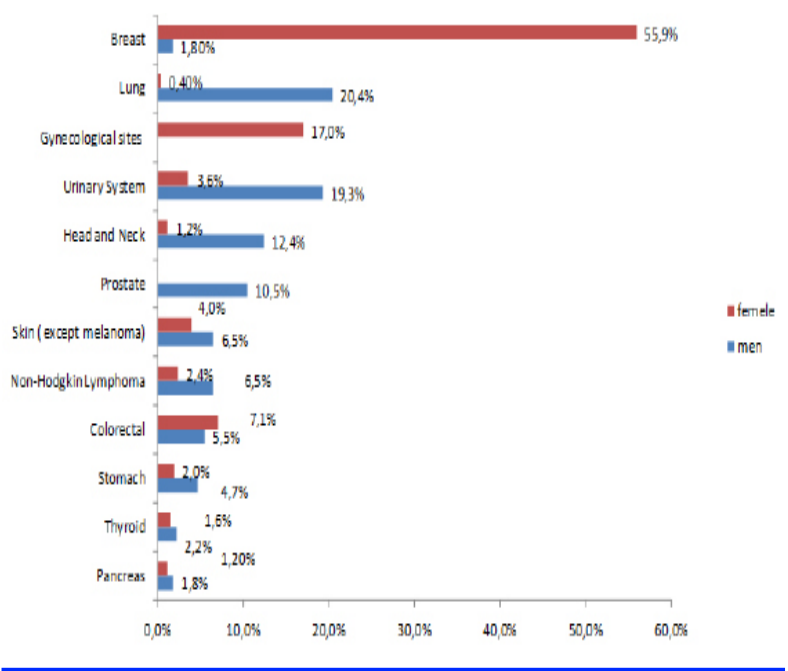

Figure 2. Distribution of Second Primary Cancer among Female Patient

was bilateral breast cancers ( 110 patients, $34.3 \%$ ) and in males, prostate-urinary tract (31 patients $21.4 \%$ ). Conversely, MPM, were in females most frequently, breast and gynecologic cancer (19 patients, 24.3\%) and lung and head and neck cancer (14 patients, $11.6 \%$ ) in males.

\section{Discussion}

We report here in a Tunisian retrospective study, arising from the North Tunisia Cancer population-based Registry, concerning 528 cases of MPM's representing $1 \%$ of the cancer cases diagnosed during 10 years from 2000-2009. We observed that breast was the most frequent first tumor site with MPM in females and the most frequent malignant association was breast-breast cancers. In males, the most frequent MPM's occur in the urinary tract and the most common cancer associations were urinary tract and prostate cancers. Diagnosis of the primary tumor was found to be existed earlier in women than in men. In our patients, most of the tumors were diagnosed at an advanced stage (stage 3-4) and at 6 decades. Further,

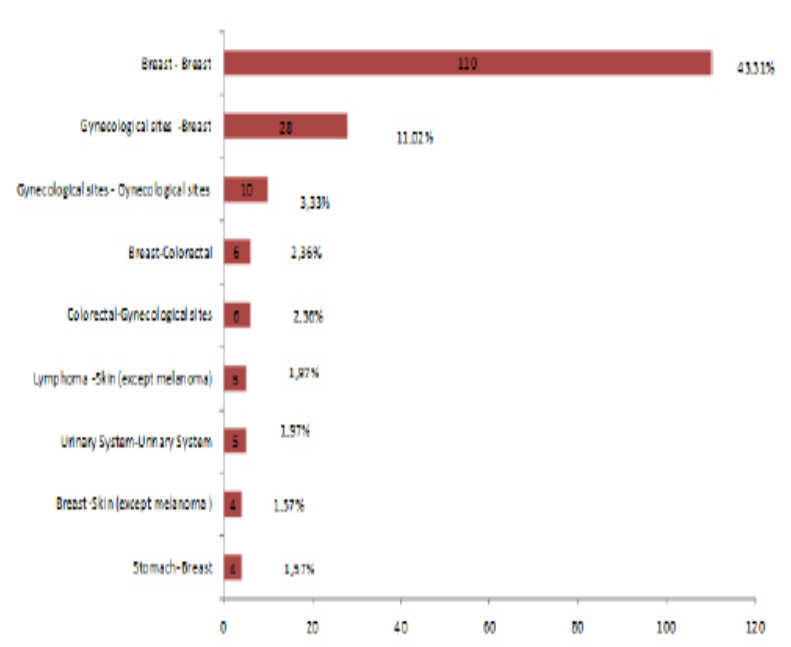

Figure 3. Most Common Cancers Associations in Female

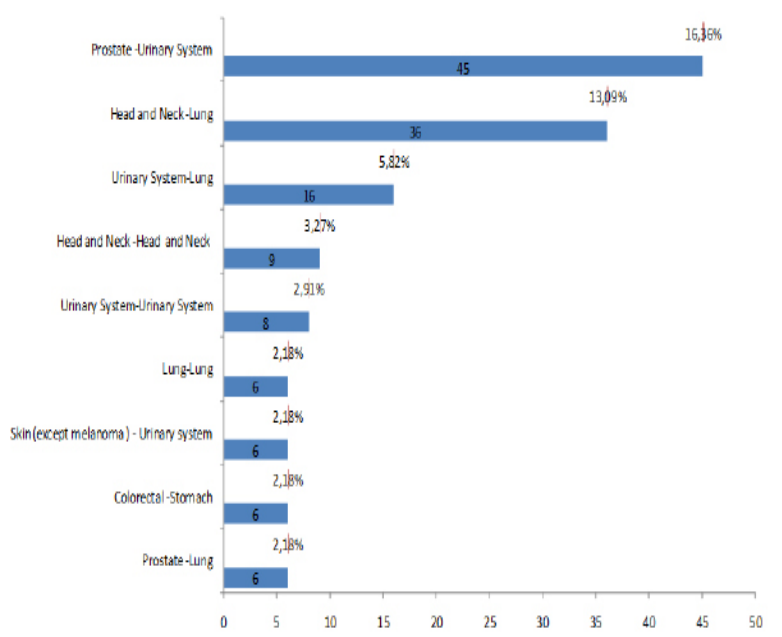

Figure 4 . Most Common Cancers Associations in Male

MPM was more often synchronous than metachronous in our result. According to the literature review, it was concluded the prevalence of MPM arranged from $0.7 \%$ to $11.3 \%$ [4-8-11]. In our studies, the overall proportion of MPM is $1 \%$ which is similar to the prevalence in China but less than reported .in other countries, [6-12]. There are several reasons for this lower proportion.1. Misdiagnosis: Physicians neglected the search of MPMT, and some patients who suffer from MPMTs may be misdiagnosed with metastatic cancer. On the other hand, On the other hand, some patients may develop MPMTs during the progression of their primary cancer but fail to see their physician [11]. The difficulty of detection: primary cancer may be very small and difficult to detect at the time of presentation. Furthermore, autopsies are rarely performed, which can also result in misdiagnosis.3.short survival time. In our study, a high proportion of patients were diagnosed at a late stage, which is correlated to short time survival and then a low probability to develop second cancer. In a review of the literature regarding MPM, the most frequently reported factors to be associated with a second primary cancer are y genetic susceptibility, longer

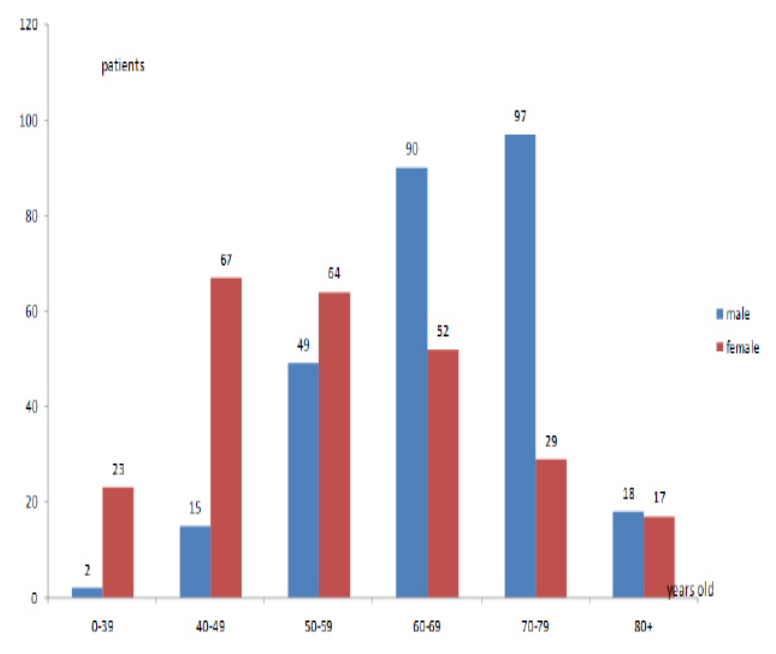

Figure 5. Distribution of First Primary Cancer According to Age 
average lifespan or medical advances in chemotherapy and radiotherapy [6-13-14]. These factors explained the high proportion of MPM reported by Yamamoto [15] (15-20\%) for Japanese patients with colorectal carcinoma. On the other hand, Carcinogenic, such as tobacco and alcohol, may increase the risk of MPM [16-18]. With the improvement of medical techniques and extended lifespan, the incidence of MPM had been increasing worldwide and been of great concern with public health [12]. Regarding the comparison of the two genders. In our studies, there was no difference between male and female; the male: female ratio was 1.08; however male predominance for MPM was generally reported. The male: female ratio ranged from 0.9: 1 to $3.5: 1$ [19-20].

According to age, in our study, the median age of the patients at the initial diagnosis was 62 ; which is consistent with the results reported while analyzing autopsy series and clinical reports, and where the median age of $50-94 \%$ of MPMN patients was over 50 years [6]. Overall, patients with MPMNs tend to be older than those with a single primary malignant neoplasm [6-8-9]; however, The lower age of women with MPMNs in comparison to men observed in our study is consistent of the result reported in Alexandru study with the highly significant statistical difference between the average age at which the first tumors occurred in the two gender groups [1].

Regarding the most frequent first cancer location associated with MPMNs, breast cancer was the most frequent first and second tumor among females in the present study; this result is consistent with those reported in several studies [4-8-11-20]. On the other hand, a high frequent association breast/breast cancer was observed. According to Chirila, the risk for women with unilateral breast cancer of developing a second primary is 2-6 times the risk in the general population of developing an initial breast cancer [4-20-21].

For women suffered from first primary breast cancer associated with MPMNs in our study, gynecologic and colorectal cancers were the second and third common cancers; this result was also reported by Babacan and al [9] and Zhihe and al [11] and This result could be explained by hormonal and nutritional factors reciprocal associations between cancers of colon, breast and female genital organs [9].

In this study, the most common tumors in men were related to smoking (head and neck, lung, and urinary tract carcinoma). It's highly probable that the same carcinogenic effects of tobacco and alcohol that give rise to the primary tumor also promote the growth of secondary tumors located in the same "condemned mucosa" of the aerodigestive tract and bladder [4-9]. It is a known fact that continued smoking increases the second cancer frequency and quitting decreases the risk [16].

The main cancers associations in men observed in our study were prostate-urinary tract, head-neck tumors-lung carcinoma in male and breast carcinoma-breast carcinoma, breast carcinoma-gynecologic cancers in females; while in the SEER [22] data the most common tumor pairs in males were prostate carcinoma-lung carcinoma and respiratory system cancers, and secondly prostate-carcinoma- colon carcinoma. In the female, the first two were breast carcinoma-breast carcinoma and breast carcinoma- colon carcinoma pairs

For the inter-tumor intervals, our observation showed that the median interval time is 1.98 months, $60.8 \%$ of patient were diagnosed as synchronous MPMN and $39.2 \%$ as metachronous MPMN. Among different publications, Babacan et al., [9] and Arpaci et al [4] reported that a median interval was 15 months and 4.65 years, respectively. With a follow-up of 20 years and 30 years respectively, the periods of the two studies are much longer than the 10 years of our study. It can explain that we have many synchronous cancers than metachronous. Arpaciet al. reported $34 \%$ of synchronous and $66 \%$ of metachronous tumors in their MPMN series [4]. Babacan reported $18,4 \%$ of the patients were diagnosed with synchronous and $81.6 \%$ with metachronous MPMNs [9] the most frequently detected synchronous tumors were bilateral breast cancers and prostate-urinary tract respectively in female and in the male. In other publication, Synchronous MPMNs are often seen in the genitourinary and gastrointestinal system [6] metachronous MPMN are lung cancer and breast cancer [9]. Of Synchronous MPM patients, most cases have occurred at the same time [8]. However, in Tunisia, Long delay consultation time remains a determining factor for the late diagnosis of breast cancer [23], widely observed, in the Tunisian patient. The latter poses real problems of organization and means in the care of the patients.

Considering the cancer stage at diagnosis, in our study, both primary and secondary tumors tended to be in an advanced stage. The same result was also reported in some studies [1-4]. It should be explained either by the low compliance of patients to follow-up or by their tendency to neglect symptoms. Therefore, doctors should give warnings regarding the risk of developing secondary malignancies after the primary treatment and also about the occurrence of any new symptoms [4-24].

There were some limitations in our study. First, due of a long delay for patients to consult their physician, the interval between first and second cancer could be largely influenced by the behavior of patients to consult and also the physician to search a second cancer Second, due to the poor compliance of our patients to follow-up, a survival analysis was not possible.

In our study, $1 \%$ of the patients were diagnosed with MPMN. Most common malignancy associations in MPM were the prostate-urinary tract, head-neck tumors-lung carcinoma in male and breast carcinoma-breast carcinoma, breast carcinoma-gynecologic cancers in females. As main recommendations, taking into consideration the risk of second cancer, it is important that patients treated for cancer should be followed for a long time. Each patient must be informed about the risk of developing secondary malignancies after the first treatment and about the importance of reporting any new symptom which might occur.

Careful monitoring ensures an early detection of secondary tumors, and, subsequently, an appropriate management. Nonetheless, cancer patients should be 
informed about prevention (cessation of smoking and alcohol use, protection from UV light etc.), screening tests (mammography, cervical smear, fecal occult blood test etc.) and should be encouraged to modify their lifestyle.

\section{References}

1. Irimie A A-CP, Burz C, Puscas E Multiple primary malignancies-epidemiological analysis at a single tertiary institution. . J Gastrointestin Liver Dis,. (2010 ). ;19 $((1)):, 69-73$. .

2. Narendra H SR, Joseph T, Donald JF Second Primary Malignant Neoplasms: A Clinicopathological Analysis from a Cancer Centre in India. . Asian Pacific J Cancer Prev ,. (2012).13 ((12),):6087-91.

3. Travis LB, Demark Wahnefried W, Allan JM, Wood ME, $\mathrm{Ng}$ AK. Aetiology, genetics and prevention of secondary neoplasms in adult cancer survivors. Nature reviews Clinical oncology. 2013;10(5):289-301.

4. Arpaci E, Tokluoglu S, Yetigyigit T, Alkis N. Multiple primary malignancies--a retrospective analysis at a single center in Turkey. Asian Pacific journal of cancer prevention : APJCP. 2013;14(2):769-73.

5. Warren S GO. Multiple primary malignant tumors: A survey of the literature and statistical study. Am J Cancer,. (1932). $; 16,: 1358-414$.

6. Meng L XZ, Yanwei S, et al Clinical analysis and prognosis of synchronous and metachronous multiple primary malignant tumors. Medecine (Baltimore), . (2017). ;96((17),):e6799.

7. International rules for multiple primary cancers. Asian Pacific journal of cancer prevention : APJCP. 2005;6(1):104-6.

8. Feng J L-JY, Jin Z, Hai H, Li-Wei W. Clinical Features of Multiple Primary malignancies: a Retrospective Analysis of 72 Chinese Patients. . Asian Pac J Cancer Prev,. (2014 ) .15 ((1), ):331-4.

9. Babacan N.A AS, Cetin B, et al Multiple primary malignant neoplasms: Multi-center results from Turkey. Journal of BUON,. (2012 ). 17,:770-5.

10. Morris LG, Sikora AG, Hayes RB, Patel SG, Ganly I. Anatomic sites at elevated risk of second primary cancer after an index head and neck cancer. Cancer causes \& control : CCC. 2011;22(5):671-9.

11. Zhihe L CL, Wei G, Siyun L, Ou B Clinical Analysis of 152 Cases of Multiple Primary A. (2015) .

12. Chang CC, Chung YH, Liou CB, Lee YC, Weng WL, Yu $\mathrm{YC}$, et al. Influence of residential environment and lifestyle on multiple primary malignancies in Taiwan. Asian Pacific journal of cancer prevention : APJCP. 2015;16(8):3533-8.

13. Hu NC HS, Chen TJ, et al Multiple primary malignancies including colon, stomach, lung, breast, and liver cancer: a case report and literature review. . Chin Med J (Eng), (2009). ;122,:3091-3.

14. Angurana SL, Kapoor R, Kumar P, Khosla D, Kumar N, Sharma SC, et al. Quadruple malignancy in a single patient: a case report and comprehensive review of literature. Journal of cancer research and therapeutics. 2010;6(2):230-2.

15. Yamamoto S, Yoshimura K, Ri S, Fujita S, Akasu T, Moriya Y. The risk of multiple primary malignancies with colorectal carcinoma. Diseases of the colon and rectum. 2006;49(10 Suppl):S30-6.

16. Sang Min PB TL, Shaowei WD, et al. Risk of second primary cancer associated with pre-diagnostic smoking, alcohol, and obesity in women with keratinocyte carcinoma. . Cancer Epidemiology,. (2017). ;47,:106-13.

17. Feng Z, Xu QS, Niu QF, Qin LZ, Li JZ, Su M, et al. Risk factors for patients with multiple synchronous primary cancers involving oral and oropharyngeal subsites. Oral surgery, oral medicine, oral pathology and oral radiology. 2016;121(4):360-6.

18. Braisch U, Meyer M, Radespiel-Troger M. Risk of tobaccorelated multiple primary cancers in Bavaria, Germany. BMC cancer. 2012;12:250.

19. Gursel B, Meydan D, Ozbek N, Ozdemir O, Odabas E. Multiple primary malignant neoplasms from the black sea region of Turkey. The Journal of international medical research. 2011;39(2):667-74.

20. Gulhan I ES, Yakut C, et al. Second primary gynecologic cancers after breast cancer in Turkish women. . Int J Gynecol Cancer, . (2009). ;19,:648-50. .

21. Chirila DN, Turdeanu NA, Constantea NA, Coman I, Pop T, Popp RA, et al. Multiple malignant tumors. Chirurgia (Bucharest, Romania : 1990). 2013;108(4):498-502.

22. Hayat MJ, Howlader N, Reichman ME, Edwards BK. Cancer statistics, trends, and multiple primary cancer analyses from the Surveillance, Epidemiology, and End Results (SEER) Program. The oncologist. 2007;12(1):20-37.

23. Landolsi A, Gahbiche S, Chaafii R, Chabchoub I, Ben Fatma L, Hochlef M, et al. [Reasons of diagnostic delay of breast cancer in Tunisian women (160 patients in the central region of Tunisia)]. La Tunisie medicale. 2010;88(12):894-7.

24. Luciani A, Ascione G, Marussi D, Oldani S, Caldiera S, Bozzoni S, et al. Clinical analysis of multiple primary malignancies in the elderly. Medical oncology (Northwood, London, England). 2009;26(1):27-31.

\section{(1) (3)}

This work is licensed under a Creative Commons AttributionNon Commercial 4.0 International License. 